\title{
Near-IR spectroscopy of planetary nebulae precursors ${ }^{\star}$
}

\author{
D. A. García-Hernández ${ }^{1}$, A. Manchado ${ }^{1,2}$, P. García-Lario ${ }^{3}$, C. Domínguez-Tagle ${ }^{1}$, \\ G. M. Conway ${ }^{4}$, and F. Prada ${ }^{5}$
}

1 Instituto de Astrofísica de Canarias, 38205 La Laguna, Tenerife, Spain

2 Consejo Superior de Investigaciones Científicas, CSIC

3 ISO Data Centre, Science Operations and Data Systems Division, Research and Scientific Support Department of ESA, VILSPA, 28080 Madrid, Spain

4 Department of Physics and Astronomy, University of Calgary, Alberta T2N 1N4, Canada

5 Centro Astronómico Hispano-Alemán, Apartado de Correos 511, 04080 Almería, Spain

Received 31 July 2001 / Accepted 18 March 2002

\begin{abstract}
We present near-IR spectroscopy of a sample of 30 IRAS sources recently identified as late AGB stars, post-AGB stars or early PNe. The spectra obtained are centered at various wavelengths covering the molecular hydrogen $v=1 \rightarrow 0 \mathrm{~S}(1) 2.122 \mu \mathrm{m}$ and $v=2 \rightarrow 1 \mathrm{~S}(1) 2.248 \mu \mathrm{m}$ emission lines, the recombination lines of hydrogen $\operatorname{Br} \gamma 2.166 \mu \mathrm{m}, \operatorname{Pf} \gamma 3.741 \mu \mathrm{m}$ and $\operatorname{Br} \alpha 4.052 \mu \mathrm{m}$, and the $\mathrm{CO}[v=2 \rightarrow 0]$ first overtone bandhead at $2.294 \mu \mathrm{m}$. As a result of these observations we have detected molecular hydrogen emission for the first time in 9 of these sources and confirmed a previous detection by Weintraub et al. (1998). This increases from 4 to 13 the total number of proto-PNe detected in molecular hydrogen. In most cases, the positive detections also show emission in the recombination lines of hydrogen (with the exception of IRAS 17150-3224) indicating that the onset of molecular hydrogen emission takes place in the post-AGB phase, very shortly before the nebula becomes ionized. When the molecular hydrogen is fluorescence-excited the detection rate is found to be directly correlated with the evolutionary stage of the central star, rather than with the nebular morphology. When the temperature of the central star is hot enough, fluorescence excitation can be induced by the absorption of UV photons escaping from the rapidly evolving central post-AGB star. In contrast, shock-excited molecular hydrogen is detected only in strongly bipolar proto-PNe, sometimes even at an early stage in the post-AGB phase. Shock excitation is the consequence of the interaction of the fast post-AGB wind with the slow wind material ejected during the AGB. The strong correlation of shock-excited molecular hydrogen emission with bipolarity found confirms the result previously reported by Kastner et al. (1996) in evolved PNe. However, our results show that this correlation does not exist in the case of fluorescence-excited molecular hydrogen.
\end{abstract}

Key words. stars: AGB and post-AGB - planetary nebulae: general - stars: evolution - infrared: stars stars: circumstellar matter - stars: mass loss

\section{Introduction}

The short transition phase between the end of the asymptotic giant branch (AGB) and the formation of a new planetary nebula $(\mathrm{PN})$ is still poorly understood. The main reason for this is probably twofold. First, only a small number of objects are known in this phase, as this is a short-lived evolutionary stage. Second, in many cases, stars evolve through this phase heavily obscured by the

Send offprint requests to: D. A. García-Hernández, e-mail: agarcia@ll.iac.es

* Based on observations collected at the European Southern Observatory (La Silla, Chile). strong circumstellar envelopes formed during the previous AGB phase, thus making observations difficult.

However, this is an evolutionary stage when crucial changes occur that completely determine the subsequent evolution of the individual star as a PN. Eventually, this also results in changes in the overall chemical evolution of the Galaxy, since huge amounts of processed material are transferred to the interstellar medium as a consequence of the strong mass loss experienced at the very end of the AGB phase.

It is also during this short transition phase when the ionization of the gas ejected during the previous AGB phase takes place. However, it is now well established that in most cases only a small fraction of it gets eventually 
ionized in comparison with the total amount of mass expelled by the central star during the AGB phase (tenths of a solar mass of ionized gas compared to up to several solar masses ejected during the AGB phase in the case of the more massive progenitors) (Pottasch 1980, 1992; Kwok 1994). Most of this material remains neutral in the form of dust grains, molecules or atoms which can be detected easily at infrared or radio wavelengths even when the star has completely evolved and turned into a PN.

From the analysis of IRAS data, and especially with the advent of the higher quality ISO data, it has been possible to confirm the presence of large amounts of dust grains which emit thermally in the mid- and far-infrared in objects evolving from the AGB to the PN stage. Through the analysis of the features observed in the ISO spectra it is possible to determine the dominant gas and dust chemistry (O-rich or C-rich) in the stellar photosphere and in the circumstellar envelope (Waters et al. 1999; Sylvester et al. 1999; Hrivnak et al. 2000).

The detection of molecules in the envelopes around PNe has also been successful at submilimeter and radio wavelengths over the last two decades. Strong CO molecular emission has been detected in a large number of C-rich PNe (Huggins \& Healy 1989; Huggins et al. 1996) and is also detectable around both C-rich and O-rich postAGB stars (Likkel et al. 1991; Loup et al. 1993; Neri et al. 1998, and references therein). Other molecules, like $\mathrm{HCN}$ or $\mathrm{CN}$ are detected only in envelopes dominated by a C-rich chemistry (Lindqvist et al. 2000), while in the case of circumstellar envelopes dominated by an O-rich chemistry strong molecular maser emission from $\mathrm{SiO}, \mathrm{H}_{2} \mathrm{O}$ and $\mathrm{OH}$ is usually detected when the star is in the AGB phase (Engels 1979; Engels \& Lewis 1996; Nyman et al. 1998; te Lintel Hekkert 1991a; te Lintel Hekkert et al. 1991b). In many cases, the $\mathrm{OH}$ maser emission remains detectable beyond the AGB throughout the whole post-AGB evolution (te Lintel Hekkert \& Chapman 1996) and sometimes even when the object has already become an ionized PN (the socalled OHPNe - Zijlstra et al. 1989, 1991). More complex molecules are difficult to detect, but in the circumstellar envelopes of a few objects like IRC+10216, AFGL 618 and AFGL 2688 more than 50 different molecular species have been identified in the last few years, and the number and complexity of the new molecules found is continuously increasing (Wallerstein \& Knapp 1998; Bujarrabal et al. 1988; Cernicharo et al. 1999).

Since hydrogen is the main constituent of stellar atmospheres one would expect to find also a significant amount of neutral material locked up in the form of molecular hydrogen around stars evolving from the AGB phase to the PN stage (both O-rich and C-rich). Following the first detection of molecular hydrogen in the PN NGC 7027 (Treffers et al. 1976), more than 70 other PNe have been detected in the last few years by Webster et al. (1988), Zuckerman \& Gatley (1988), Kastner et al. (1996), Latter et al. (1995), Hora et al. (1999) and Guerrero et al. (2000). As a general trend, it is found that molecular hydrogen emission is predominantly detected in type I bipolar PNe, which are expected to be the result of the evolution of the more massive AGB progenitors. It remains unclear, however, whether this is just an observational selection effect.

Very few attempts have been made so far to search for molecular hydrogen around post-AGB stars or proto-PNe, the precursors of PNe. Molecular hydrogen emission was first detected towards AFGL 618 by Beckwith et al. (1978) and AFGL 2688 by Gatley et al. (1988). More recently, Weintraub et al. (1998) looked at a small sample of five proto-PNe and detected molecular hydrogen emission in two of them (IRAS 17441-2411 and AFGL 6815S = IRAS 17150-3224), bringing to four the total number of bipolar proto-PNe with this type of emission. The discovery and study of new transition objects showing this emission at such an early stage is crucial for determining the mechanism of excitation of molecular hydrogen and the conditions needed to preserve this neutral material from destruction in more evolved PNe.

This paper extends this search to a larger sample of transition objects, including late-AGB stars, post-AGB stars with various spectral types, as well as the youngest $\mathrm{PNe}$ known, in order to look for possible correlations between the onset of molecular hydrogen emission and the evolutionary stage and/or the morphology of the sources observed.

In Sect. 2 we explain the criteria followed to select the sources in the sample. The spectroscopic observations made in the near infrared are described in Sect. 3, while the results obtained are presented in Sect. 4 and discussed in Sect. 5. The conclusions derived from these results are given in Sect. 6.

\section{Selection of the sample}

For the last ten years our group studied a large number of IRAS sources with dust temperatures similar to post-AGB stars and planetary nebulae $(\sim 100-200 \mathrm{~K})$, mainly through near-infrared photometry (García-Lario et al. 1990, 1997a; Manchado et al. 1989a) and optical spectroscopy (García-Lario et al. 1991, 1994, 1997b, 2002; Riera et al. 1995). As a result of this multi-wavelength program we have discovered heavily obscured late AGB stars and early post-AGB stars still surrounded by thick circumstellar envelopes only detectable in the near infrared as well as post-AGB stars with optically bright central stars covering all possible spectral types from $\mathrm{M}$ to $\mathrm{B}$ in what it seems to be an evolutionary sequence towards the PN stage, together with a small number of new extremely young PNe (García-Lario et al. 1997a, 2002), among them, the youngest PN known so far: the Stingray Nebula (Bobrowsky et al. 1998).

From this list of newly discovered sources, we selected a subsample of 30 , which are observable from the southern hemisphere. This subsample is also representative of the various evolutionary stages mentioned above, including very late AGB stars with no optical counterparts, post-AGB stars with all kind of spectral types and 
Table 1. IRAS sources included in the survey and main observational properties.

\begin{tabular}{|c|c|c|c|c|}
\hline IRAS name & Other names & Spectral Type & $\mathrm{H}_{\alpha}$ emission & Size/Morphology \\
\hline $05284+1945$ & & $\mathrm{OH} / \mathrm{IR}$ & - & No optical counterpart \\
\hline $05341+0852$ & & F4Iab & no & $1.1^{\prime \prime} \times 0.8^{\prime \prime} /$ Round-Elliptical \\
\hline $06530-0213$ & & F0I & no & $2.3^{\prime \prime} \times 1.0^{\prime \prime} /$ Bipolar \\
\hline $06556+1623$ & HD 51585, MWC 162 & B1I & yes (strong) & Unknown \\
\hline $07027-7934$ & & $\mathrm{PN}(\mathrm{WC} 11)$ & yes (strong) & $13^{\prime \prime} \times 15^{\prime \prime} /$ Round-Elliptical \\
\hline $07134+1005$ & SAO 96709, HD 56126 & F7I & yes (weak) & $4.7^{\prime \prime} \times 3.9^{\prime \prime} /$ Round-Elliptical \\
\hline $07331+0021$ & AI CMi, RAFGL 5236 & K3-5I & yes (weak) & Unknown \\
\hline $08005-2356$ & & F3I & yes (strong) & $2.6^{\prime \prime} \times 1.2^{\prime \prime} /$ Bipolar \\
\hline $08187-1905$ & HD 70379 & F7I & yes (weak) & Unknown \\
\hline $08242-3828$ & & $\mathrm{OH} / \mathrm{IR}$ & - & No optical counterpart \\
\hline $08425-5116$ & & $\mathrm{OH} / \mathrm{IR}$ & - & No optical counterpart \\
\hline $09024-5019$ & & - & - & No optical counterpart \\
\hline $10178-5958$ & Hen 3-401, C 1662 & B1I? & yes (strong) & $2.0^{\prime \prime} \times 14.5^{\prime \prime} /$ Bipolar \\
\hline $10197-5750$ & Roberts 22, RAFGL 4104 & $\mathrm{~A} 2 \mathrm{I}$ & yes (strong) & $7.8^{\prime \prime} \times 4.5^{\prime \prime} /$ Bipolar \\
\hline $10215-5916$ & RAFGL 4106 & G1I & yes (weak) & $\approx 7^{\prime \prime} \times 7^{\prime \prime} /$ Round-Elliptical \\
\hline $11438-6330$ & & $\mathrm{OH} / \mathrm{IR}$ & - & No optical counterpart \\
\hline $12067-4508$ & RU Cen, SAO 223245 & G2w & yes (weak) & Unknown \\
\hline $12175-5338$ & SAO 239853 & F2I & yes (weak) & Unknown \\
\hline $13421-6125$ & & $\mathrm{OH} / \mathrm{IR}$ & - & No optical counterpart \\
\hline $14104-5819$ & & $\mathrm{OH} / \mathrm{IR}$ & - & No optical counterpart \\
\hline $14331-6435$ & Hen $3-1013$ & B8I & yes (strong) & Unknown \\
\hline $16342-3814$ & & M4 & no & $2.6^{\prime \prime} \times 0.7^{\prime \prime} /$ Bipolar \\
\hline $16594-4656$ & & $\mathrm{~B} 7 ?$ & yes (strong) & $5^{\prime \prime} \times 11^{\prime \prime} /$ Multipolar \\
\hline $17119-5926$ & Hen 3-1357, Stingray Nebula & $\mathrm{PN}(\mathrm{O} 8 \mathrm{~V})$ & yes (strong) & $1.6^{\prime \prime} \times 1.4^{\prime \prime} /$ Bipolar \\
\hline $17149-3053$ & & M4? & no & Unknown \\
\hline $17150-3224$ & RAFGL 6815S & G2I & no & $11^{\prime \prime} \times 5^{\prime \prime} /$ Bipolar \\
\hline $17311-4924$ & Hen $3-1428$ & B8I & yes (strong) & Unknown \\
\hline $17411-3154$ & RAFGL 5379 & $\mathrm{OH} / \mathrm{IR}$ & - & No optical counterpart \\
\hline $17423-1755$ & Hen $3-1475$ & $\mathrm{~B} ?$ & yes (strong) & $3^{\prime \prime} \times 17^{\prime \prime} /$ Bipolar \\
\hline $18062+2410$ & SAO 85766, HD 341617 & $\mathrm{~B}$ & yes(strong) & Unknown \\
\hline
\end{tabular}

proto-PNe showing a variety of morphological structures (from round/elliptical to strongly bipolar).

In Table 1 we list the IRAS sources included in the study. In this table we also indicate some of the observational characteristics which are relevant for the discussion presented in Sect. 5, like their apparent optical morphology, spectral type, or the presence/absence of $\mathrm{H} \alpha$ emission in their optical spectra (all this information taken from García-Lario et al. 2002, and references therein).

\section{Observations and data reduction}

The observations were conducted in March 1993 and January 1994 with IRSPEC attached to the Nasmyth focus of the $3.6 \mathrm{~m}$ NTT telescope at La Silla, ESO. IRSPEC was a cryogenically cooled scanning grating IR spectrometer with a $58 \times 62$ pixel array from SBRC as detector (Moorwood et al. 1986). It covered the $1-5$ microns wavelength range with a spectral resolution between 1500 and 2000 . The spatial scale was $2.2^{\prime \prime} /$ pixel, the slit width was $4.5^{\prime \prime}$ and the un-vignetted field was $\sim 100^{\prime \prime}$. The array had a quantum efficiency of 0.89 at $2.85 \mu \mathrm{m}$, and a well capacity of $10^{6} \mathrm{e}^{-}$. This allowed exposures up to $60 \mathrm{~s}$ per frame in the $2-2.6 \mu \mathrm{m}$ region. For integration times around $10 \mathrm{~s}$, the combined dark and read noise was $\sim 150 \mathrm{e}^{-}$. Owing to the poor wavelength coverage of the spectrograph in a single exposure, different exposures were needed centered at various wavelengths, each one covering a different spectral feature under analysis. The lines observed were the molecular ro-vibrational lines of molecular hydrogen $v=1 \rightarrow 0 \mathrm{~S}(1) 2.122 \mu \mathrm{m}$ and $v=2 \rightarrow 1 \mathrm{~S}(1) 2.248 \mu \mathrm{m}$, the recombination lines of hydrogen $\operatorname{Br} \gamma 2.166 \mu \mathrm{m}, \operatorname{Pf} \gamma$ $3.741 \mu \mathrm{m}$ and $\operatorname{Br} \alpha 4.052 \mu \mathrm{m}$ and the $\mathrm{CO}[v=2 \rightarrow 0]$ $2.294 \mu \mathrm{m}$ first overtone bandhead. Total on-source integration times were typically of $240 \mathrm{~s}$ with exposures ranging from $36 \mathrm{~s}$ to $1440 \mathrm{~s}$ in the most extreme cases.

The slit orientation was north-south $\left(\mathrm{PA}=0^{\circ}\right)$ in 1993 with the exception of IRAS 10178-5958 (PA = $\left.70^{\circ}\right)$, IRAS $16594-4656\left(\mathrm{PA}=70^{\circ}\right)$, IRAS 16342-3814 $\left(\mathrm{PA}=-70^{\circ}\right)$ and IRAS 17423-1755 $\left(\mathrm{PA}=+55^{\circ}\right)$, while in 1994 the slit orientation was always east-west $\left(\mathrm{PA}=90^{\circ}\right)$. The changes in the slit orientation during the 1993 run were in principle made in order to follow the bipolar axis of some well known extended objects, like the ones listed above. However, due to a mistake in the determination of the sign, IRAS 17423-1755 was observed almost perpendicular to the actual bipolar axis in 1993 while in IRAS 16342-3814 independently of the slit orientation, we cover the source completely. The only other known extended bipolar objects in our sample are IRAS 10197-5750 and IRAS 17150-3224. 
Table 2. Summary table with the results obtained. Quoted values are the emission line fluxes or $2 \sigma$ upper limits to the expected emission line fluxes (in units of $10^{-14} \mathrm{erg} \mathrm{cm}^{-2} \mathrm{~s}^{-1}$ ). The equivalent widths (in units of $10^{-4} \mu \mathrm{m}$ ) are shown in brackets for the features detected in absorption.

\begin{tabular}{|c|c|c|c|c|c|c|c|c|c|c|c|c|}
\hline \multirow{2}{*}{$\frac{\text { IRAS name }}{05284+1945}$} & \multicolumn{2}{|c|}{$\mathrm{H}_{2}[v=1 \rightarrow 0 \mathrm{~S}(1)]$} & \multicolumn{2}{|c|}{$\operatorname{Br} \gamma$} & \multicolumn{2}{|c|}{$\mathrm{H}_{2}[v=2 \rightarrow 1 \mathrm{~S}(1)]$} & \multicolumn{2}{|c|}{$\mathrm{CO}[v=2 \rightarrow 0]$} & \multicolumn{2}{|c|}{$\mathrm{Pf} \gamma$} & \multicolumn{2}{|c|}{$\operatorname{Br} \alpha$} \\
\hline & $\ldots$ & & n.d. & $\leq 1.3$ & $\ldots$ & & $\ldots$ & & $\ldots$ & & $\ldots$ & \\
\hline $05341+0852$ & $\mathrm{c}$ & $\leq 0.7$ & $\mathrm{~A}$ & {$[2.2]$} & $\ldots$ & & $\mathrm{c}$ & $\leq 1.4$ & $\ldots$ & & $\ldots$ & \\
\hline $06530-0213$ & c & $\leq 1.0$ & $\mathrm{~A}$ & {$[2.1]$} & $\ldots$ & & $\ldots$ & & $\ldots$ & & n.d. & $\leq 38.9$ \\
\hline $06556+1623$ & $\mathrm{E}$ & 3.4 & $\mathrm{E}$ & 63.2 & $\mathrm{c}$ & $\leq 2.0$ & $\mathrm{c}$ & $\leq 4.1$ & $\mathrm{E}$ & 13.0 & $\ldots$ & \\
\hline $07027-7934$ & $\mathrm{E}$ & 2.4 & $\mathrm{E}$ & 13.4 & $\mathrm{c}$ & $\leq 1.9$ & c & $\leq 4.0$ & $\mathrm{E}$ & 16.4 & $\mathrm{E}$ & 62.5 \\
\hline $07134+1005$ & c & $\leq 8.0$ & $\mathrm{~A}$ & {$[3.5]$} & $\ldots$ & & $\ldots$ & & $\ldots$ & & $\ldots$ & \\
\hline $07331+0021$ & c & $\leq 39.4$ & $\mathrm{c}$ & $\leq 49.4$ & $\ldots$ & & $\ldots$ & & $\ldots$ & & $\mathrm{c}$ & $\leq 97.4$ \\
\hline $08005-2356$ & c & $\leq 9.0$ & c & $\leq 13.0$ & $\ldots$ & & $\mathrm{E}$ & 24.8 & $\mathrm{c}$ & $\leq 47.5$ & $\mathrm{c}$ & $\leq 21.0$ \\
\hline $08187-1905$ & c & $\leq 4.2$ & $\mathrm{~A}$ & {$[2.4]$} & $\ldots$ & & $\ldots$ & & $\ldots$ & & $\mathrm{c}$ & $\leq 38.5$ \\
\hline $08242-3828$ & c & $\leq 30.0$ & $\mathrm{c}$ & $\leq 21.4$ & $\ldots$ & & $\ldots$ & & $\ldots$ & & c & $\leq 32.2$ \\
\hline $08425-5116$ & c & $\leq 0.5$ & c & $\leq 0.5$ & $\ldots$ & & $\mathrm{A}$ & {$[4.7]$} & $\ldots$ & & c & $\leq 17.3$ \\
\hline 09024-5019 & c & $\leq 0.4$ & $\mathrm{E}$ & 18.8 & $\ldots$ & & $\mathrm{c}$ & $\leq 0.3$ & $\mathrm{E}$ & 12.2 & $\mathrm{E}$ & 86.0 \\
\hline $10178-5958$ & $\mathrm{E}$ & $37.1^{*}$ & $\mathrm{E}$ & $25^{*}$ & $\mathrm{E}$ & 1.4 & $\mathrm{E}$ & 2.9 & $\mathrm{E}$ & 9.3 & $\mathrm{E}$ & 78.4 \\
\hline $10197-5750$ & $\mathrm{E}$ & 8.9 & $\mathrm{E}$ & 22.3 & $\mathrm{c}$ & $\leq 3.4$ & $\mathrm{c}$ & $\leq 7.1$ & $\mathrm{E}$ & 5.6 & $\mathrm{E}$ & 46.7 \\
\hline $10215-5916$ & c & $\leq 203.7$ & $\mathrm{c}$ & $\leq 198.3$ & $\ldots$ & & $\ldots$ & & $\ldots$ & & $\mathrm{E}$ & 49.1 \\
\hline $11438-6330$ & c & $\leq 0.4$ & $\ldots$ & & $\ldots$ & & $\ldots$ & & $\mathrm{c}$ & $\leq 27.5$ & $\mathrm{c}$ & $\leq 55.5$ \\
\hline $12067-4508$ & c & $\leq 3.0$ & $\mathrm{c}$ & $\leq 4.9$ & $\ldots$ & & $\ldots$ & & $\ldots$ & & $\mathrm{c}$ & $\leq 15.9$ \\
\hline $12175-5338$ & c & $\leq 1.4$ & $\mathrm{~A}$ & {$[2.7]$} & $\ldots$ & & $\mathrm{c}$ & $\leq 3.6$ & $\ldots$ & & n.d. & $\leq 18.1$ \\
\hline $13421-6125$ & c & $\leq 0.4$ & $\mathrm{c}$ & $\leq 0.3$ & $\ldots$ & & $\ldots$ & & $\ldots$ & & n.d. & $\leq 18.1$ \\
\hline $14104-5819$ & c & $\leq 1.8$ & c & $\leq 1.2$ & $\ldots$ & & $\ldots$ & & $\ldots$ & & n.d. & $\leq 17.8$ \\
\hline $14331-6435$ & $\mathrm{E}$ & 10.1 & $\mathrm{E}$ & 3.4 & $\mathrm{E}$ & 1.2 & $\ldots$ & & $\ldots$ & & n.d. & $\leq 27.2$ \\
\hline $16342-3814$ & c & $\leq 5.1$ & $\mathrm{c}$ & $\leq 4.8$ & $\ldots$ & & $\ldots$ & & $\ldots$ & & n.d. & $\leq 15.4$ \\
\hline $16594-4656$ & $\mathrm{E}$ & $62.7^{*}$ & $\mathrm{E}$ & 9.2 & $\mathrm{E}$ & 1.9 & $\mathrm{c}$ & $\leq 2.5$ & $\mathrm{c}$ & $\leq 15.3$ & $\mathrm{E}$ & 13.3 \\
\hline $17119-5926$ & $\mathrm{E}$ & 4.1 & $\mathrm{E}$ & 43.7 & $\mathrm{E}$ & 2.7 & c & $\leq 0.4$ & $\mathrm{E}$ & 9.7 & $\mathrm{E}$ & 125.5 \\
\hline $17149-3053$ & c & $\leq 3.2$ & $\mathrm{c}$ & $\leq 3.8$ & $\ldots$ & & $\ldots$ & & $\ldots$ & & $\ldots$ & \\
\hline $17150-3224$ & $\mathrm{E}$ & $5.5^{*}$ & $\mathrm{~A}$ & {$[3.1]$} & $\mathrm{c}$ & $\leq 0.7$ & $\mathrm{c}$ & $\leq 0.9$ & $\ldots$ & & $\ldots$ & \\
\hline $17311-4924$ & $\mathrm{E}$ & 36.3 & $\mathrm{E}$ & 2.0 & $\mathrm{E}$ & 3.2 & c & $\leq 1.3$ & n.d. & $\leq 8.9$ & n.d. & $\leq 14.1$ \\
\hline $17411-3154$ & c & $\leq 0.4$ & c & $\leq 0.4$ & $\ldots$ & & $\ldots$ & & $\ldots$ & & $\ldots$ & \\
\hline $17423-1755$ & c & $\leq 3.7$ & $\mathrm{E}$ & 26.2 & $\ldots$ & & $\mathrm{E}$ & 31.7 & $\mathrm{c}$ & $\leq 27.0$ & $\mathrm{E}$ & 38.1 \\
\hline $18062+2410$ & $\mathrm{E}$ & 3.0 & $\mathrm{E}$ & 0.8 & $\ldots$ & & $\mathrm{c}$ & $\leq 0.5$ & $\mathrm{c}$ & $\leq 14.2$ & n.d. & $\leq 26.7$ \\
\hline
\end{tabular}

E: emission.

A: absorption.

c: continuum.

n.d.: not detected.

...: not tried.

${ }^{*}$ : mean value of the integrated line fluxes in both runs.

IRAS 10197-5750 was only observed in 1994, when the orientation of the slit was $90^{\circ}$, while the bipolar axis of this source is located at $\mathrm{PA} \sim 20^{\circ}$. This means that the slit was positioned also very close to the direction perpendicular to its bipolar axis. In the case of IRAS 17150-3224, we observed the source both in 1993 and 1994 with orientations of 0 and $90^{\circ}$ respectively, while the actual bipolar axis of the source is now known to be located at $\mathrm{PA} \sim-45^{\circ}$. Two other sources (IRAS 10178-5958 and IRAS 16594-4656) also were observed twice. The implications of these misadjustments in the analysis of the results obtained will be discussed later.

The standard beam-switching technique was used in order to subtract the sky background. The data reduction process includes bias and flat-field corrections, as well as wavelength calibration, sky subtraction, and absolute flux calibration using reference standard stars. For this we made use of the $\mathrm{S} / \mathrm{W}$ package IRSPEC installed on MIDAS (version November 1998). 


\section{Results}

In Table 2 we list the detections/non-detections made as a result of the observations described in Sect. 3 .

All sources but one in the sample were observed in the wavelength region surrounding the $\mathrm{H}_{2}[v=1 \rightarrow 0 \mathrm{~S}(1)]$ line, resulting in positive detections in 10 cases. Out of them, 9 sources were also observed in the weaker $\mathrm{H}_{2}[v=2 \rightarrow 1$ $\mathrm{S}(1)$ ] line, among which 5 showed some emission.

The Br $\gamma$ line was detected in 17 objects, either in emission (11) or in absorption (6). In a few cases, observations were also performed in other spectral lines like $\operatorname{Pf} \gamma$, and $\operatorname{Br} \alpha$, or around the $\mathrm{CO}[v=2 \rightarrow 0]$ first overtone bandhead, in order to obtain complementary information.

The quantitative results are also shown in Table 2. Quoted values are the emission line fluxes derived by fitting gaussians to the observed line profiles or $2 \sigma$ upper limits to the expected emission line fluxes in the case of those sources with no emission detected over the underlying near infrared continuum. Both are shown in units of $10^{-14} \mathrm{erg} \mathrm{cm}^{-2} \mathrm{~s}^{-1}$. We have also estimated the equivalent widths of those features detected in absorption (in units of $10^{-4} \mu \mathrm{m}$ ). The values obtained are shown in Table 2 within brackets.

In a few cases, we did not detect any significant signal in the $2 \mathrm{D}$ spectra (nor even a continuum). These sources have been classified in Table 2 as not detected. In these latter cases we can generally infer the object's position on the $2 \mathrm{D}$ frames based on information obtained at other spectral lines and estimate the associated rms of the pixels where the feature investigated had been detected. Then we use these rms to calculate the quoted values as $2 \sigma$ upper limits to the expected emission line fluxes in Table 2 .

We estimate that for a typical $240 \mathrm{~s}$ total on-target exposure and when the underlying near infrared continuum is weak our $2 \sigma$ detection threshold is of the order of $\sim 10^{-15} \mathrm{erg} \mathrm{cm}^{-2} \mathrm{~s}^{-1}$ for the $\mathrm{H}_{2}[v=1 \rightarrow 0 \mathrm{~S}(1)]$, $\mathrm{Br}_{\gamma}, \mathrm{H}_{2}[v=2 \rightarrow 1 \mathrm{~S}(1)]$ and $\mathrm{CO}[v=2 \rightarrow 0]$ lines and of $\sim 10^{-14} \mathrm{erg} \mathrm{cm}^{-2} \mathrm{~s}^{-1}$ in the $\operatorname{Pf} \gamma$ and $\operatorname{Br} \alpha$ regions. Note that the $2 \sigma$ upper limits in Table 2 are very different from source to source because the rms is dependent of the continuum.

Three objects (IRAS 10178-5958, IRAS 16594-4656 and IRAS 17150-3224), were observed in both runs (1993 and 1994) and for them we quote in Table 2 a mean value of the integrated line fluxes (the flux differences from epoch to epoch are less than 20\%). However, the emission detected was always found to be consistent with a point-like nature of the observed source with the exception of IRAS 10178-5958 and IRAS 17119-5926, where the emission found is clearly extended (see Sect. 5.5).

Spectra for all objects (except for IRAS $05284+1945$, whose near infrared counterpart was not detected at any wavelength) in $\operatorname{Br} \gamma, \mathrm{H}_{2}[v=1 \rightarrow 0 \mathrm{~S}(1)], \mathrm{H}_{2}[v=2 \rightarrow 1 \mathrm{~S}(1)]$, $\mathrm{CO}[v=2 \rightarrow 0], \operatorname{Pf} \gamma$ and $\operatorname{Br} \alpha$ are presented in Figs. 1a to 1f. For those sources that were observed twice we show the best spectrum.

\section{Discussion}

\subsection{Hydrogen recombination lines}

In general, the strength of the near infrared hydrogen lines detected in the stars in our sample is consistent with the spectral types derived from optical data. All objects where a $\mathrm{Br} \gamma$ absorption has been detected show $\mathrm{H} \alpha$ absorption in the optical or very weak emission, while those with $\mathrm{Br} \gamma$ strongly in emission in the near infrared are also strong $\mathrm{H} \alpha$ emitters in the optical, as expected.

In the case of the late AGB stars included in our study (those classified as $\mathrm{OH} / \mathrm{IR}$ stars in Table 1) the $\mathrm{Br} \gamma$ line region is veiled by the strong dust continuum emission and we are not able to detect any signature of this hydrogen recombination line. These stars are pulsating with very long periods so heavily obscured by their circumstellar envelopes that they usually do not show any optical counterpart.

The same veiling effect might be the reason why IRAS 08005-2356 shows a featureless continuum around the $\mathrm{Br} \gamma$ line as well as at other near infrared hydrogen recombination lines, while in the optical a relatively strong $\mathrm{H} \alpha$ emission is detected. Another possibility is that the emission is just strong enough to fill in the photospheric absorption and, thus, both components compensate each other, leading to a flat continuum.

IRAS 09024-5019 seems to be also a remarkable case, since it shows a clear emission in the light of $\operatorname{Br} \gamma$ and $\operatorname{Br} \alpha$ while it does not have any (known) optical counterpart. This star could be a heavily obscured transition object in which the onset of the ionization is now taking place.

\subsection{CO first overtone}

The effective temperature of late AGB stars is expected to be very low $(\leq 2500 \mathrm{~K})$. Consistent with this, a strong $\mathrm{CO}$ photospheric absorption was detected in IRAS $08425-5116$ at $2.29 \mu \mathrm{m}$, the only late AGB star included in our sample for which we obtained a spectrum at this wavelength.

This feature corresponds to the $\mathrm{CO}$ first overtone bandhead, which is expected to be visible in stars with effective temperatures below $5000 \mathrm{~K}$. However, IRAS 17150-3224, a post-AGB star with a cool central star (G2I), did not show any signature of this bandhead, probably because of the veiling produced by the strong thermal emission from the hot dust formed in the circumstellar envelope, which completely dominates the observed spectral energy distribution at this wavelength.

Noteworthy, in three of the post-AGB stars with the earlier spectral types in the sample we detected the $\mathrm{CO}$ first overtone bandhead in emission. This has also been observed in a few other transition objects before (Oudmaijer et al. 1995) and it has been interpreted as the result of active post-AGB mass loss, the CO lines being formed in the dense outflow. 

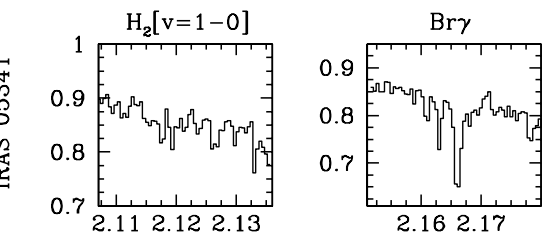

$\mathrm{H}_{2}[\mathrm{v}=2-1]$

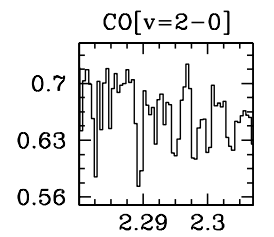

Pf $\gamma$
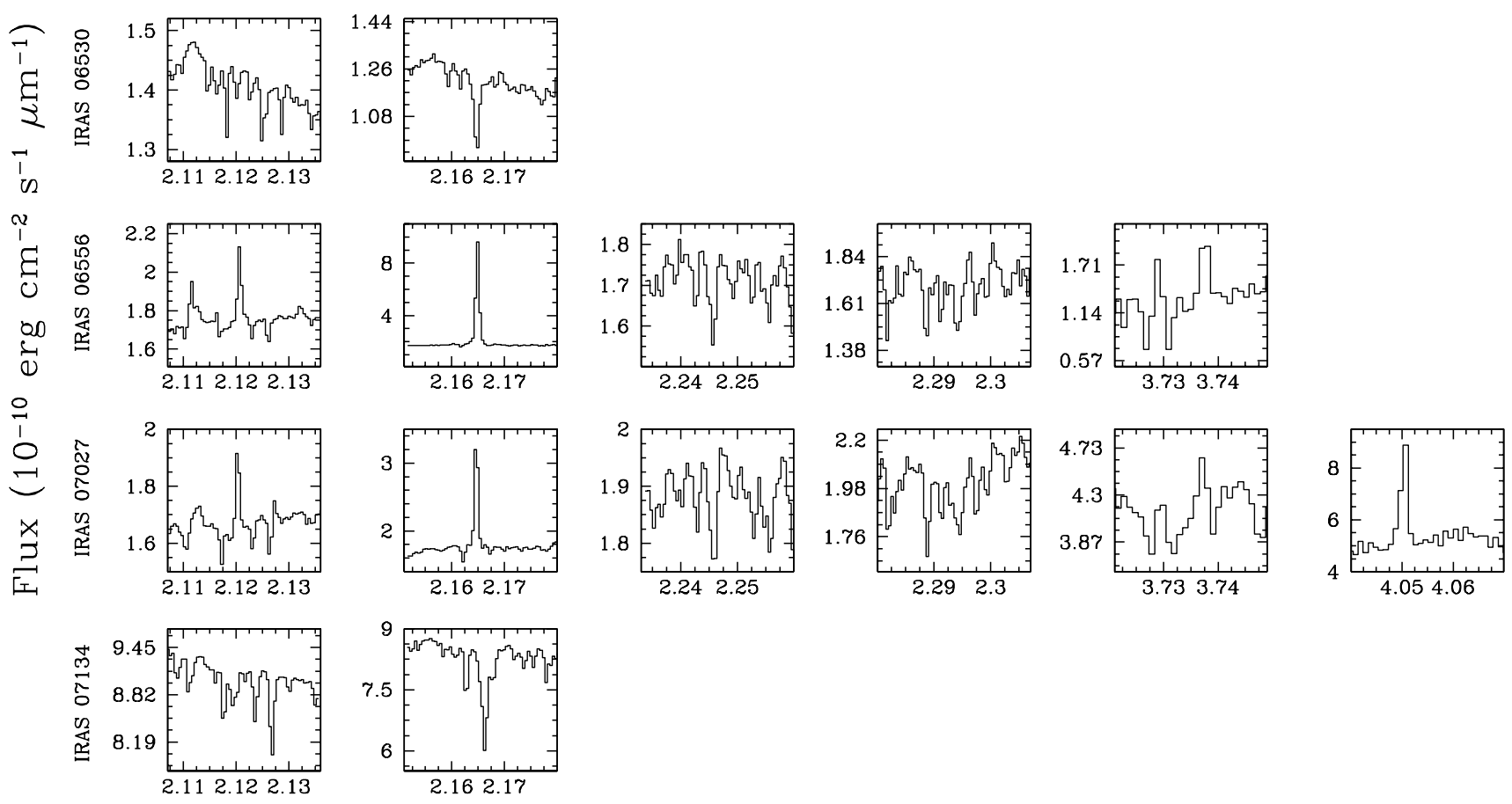

\section{Wavelength $(\mu \mathrm{m})$}

Fig. 1. a) Near infrared spectra of the sources detected with IRSPEC. Each row corresponds to observations of a different object. The object name is shown on the left along the vertical direction and they are displayed in increasing RA order

\section{3. $\mathrm{H}_{2}$ emission and evolutionary stage}

In order to understand the mechanism of excitation of molecular hydrogen it is worth to try to find a possible correlation between the frequency of positive detections and the evolutionary stage (spectral type of the central star) of the sources observed.

Starting from the less evolved objects, we find that none of the late-AGB stars included in the sample show any indication of the presence of $\mathrm{H}_{2}$ emission at $2.122 \mu \mathrm{m}$. It is important to note however that, as for the hydrogen recombination lines above discussed, the spectral region around this molecular hydrogen line appears usually completely dominated by the thermal emission coming from the hot dust in the envelope, making the detection of any spectral feature difficult in this kind of objects.

More interesting is the fact that, with the only exception of IRAS 17150-3224, which will be discussed below, none of the post-AGB stars in the sample with spectral types later than A showed any detectable emission in this line. This includes $6 \mathrm{~F}$-type, 2 G-type, $1 \mathrm{~K}$-type and $1 \mathrm{M}$ type post-AGB stars. It should be noticed that in these optically bright post-AGB stars the veiling effect from the circumstellar dust is small and cannot justify the nondetections.

In contrast, all the stars identified as post-AGB candidates in our list with spectral types earlier than A were detected in molecular hydrogen at $2.122 \mu \mathrm{m}$. In this case, the only exception was IRAS $17423-1755$, whose central star has tentatively been classified as A-type or B-type and it shows no evidence of $\mathrm{H}_{2}$ emission at $2.122 \mu \mathrm{m}$. If this emission comes mainly from the bipolar lobes, it may have not been covered by our slit because it was positioned by mistake perpendicular to the bipolar axis (see Sect. 3). Consistently, we did not detect any extended emission from this object in any other spectral line (nor even in $\operatorname{Br} \gamma$ which is detected very strong in emission). In addition, this is also a quite peculiar, extended bipolar proto-PN which shows a very high velocity field and a strong near infrared excess (Riera et al. 1995; GarcíaLario et al. 1997a). Thus, the $\mathrm{H}_{2}$ emission may be veiled by the strong underlying continuum and/or the molecular hydrogen molecules may have been destroyed by the strong stellar winds.

Out of the 10 objects in which we detected molecular hydrogen emission, 9 are new detections (only 

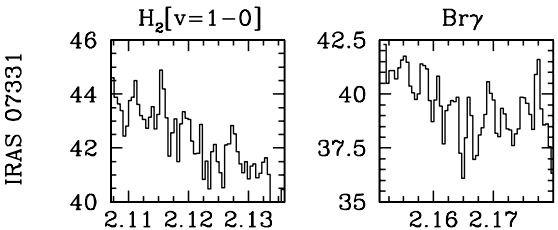

$\mathrm{H}_{2}[\mathrm{v}=2-1]$

$\mathrm{CO}[\mathrm{v}=2-0]$

Pf $\gamma$
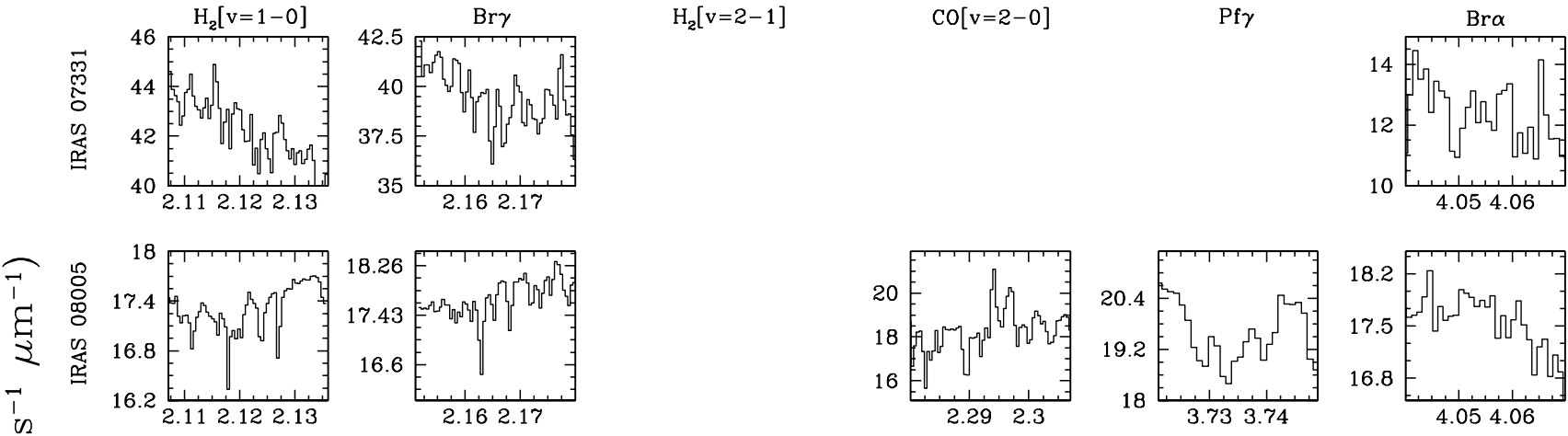

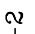
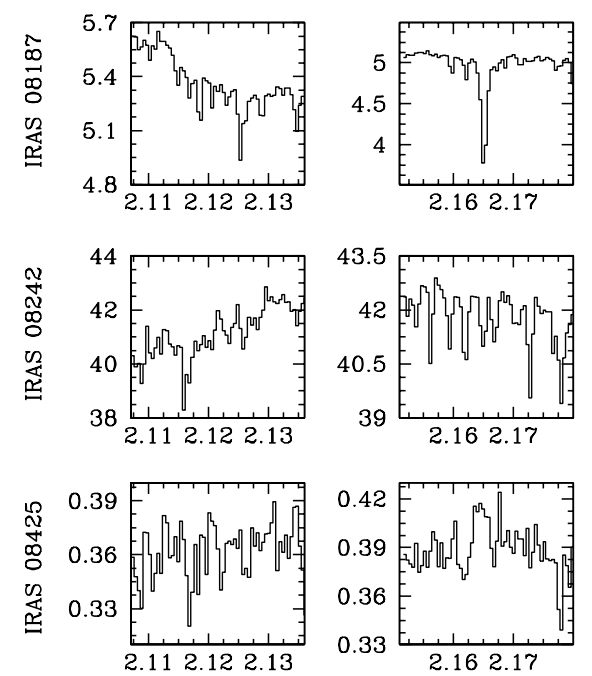
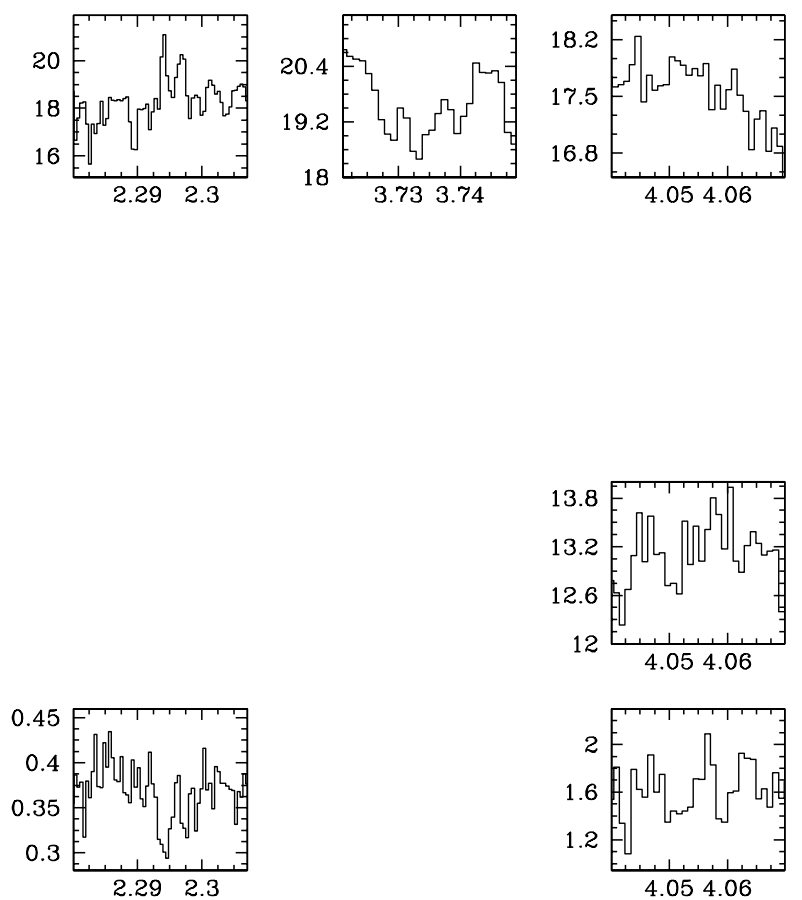

\section{Wavelength $(\mu \mathrm{m})$}

Fig. 1. b) Near infrared spectra of the sources detected with IRSPEC (continued).

IRAS 17150-3224 was previously reported by Weintraub et al. 1998). This brings the total number of transition stars in which molecular hydrogen emission has been detected from 4 to 13 . Note that the $\mathrm{H}_{2}$ emission in IRAS 10178-5958 was previously presented by us (García-Lario et al. 1999b).

The results above are consistent with the nondetection of molecular hydrogen emission in the bipolar proto-PNe $\mathrm{OH} 231.8+4.2$, IRAS $07131-0147$ or IRAS $09371+1212$ by Weintraub et al. (1998), all of which have late-type $\mathrm{M}$ central stars.

However, IRAS $17150-3224$ is not the only post-AGB star with a spectral type later than A showing $\mathrm{H}_{2}$ emission in the short list of known $\mathrm{H}_{2}$ emitters. There is another discrepant case in the literature, the well-known PPN CRL 2688 (Sahai et al. 1998), whose central star is classified as F5Iae, although it is suspected to be a binary.

\section{4. $\mathrm{H}_{2}$ emission and morphology}

It has been suggested that molecular hydrogen emission is a signpost for bipolarity in evolved $\mathrm{PNe}$ (Kastner et al. 1996). In order to explore the validity of this statement also in the precursors of PNe we can search for a possible correlation between the detection rate of molecular hydrogen emission and the morphology of the sources included in our sample.

For this, we will restrict our analysis in the following to those sources for which morphological information exists. This means that we will not consider, of course, any of the heavily obscured objects in the list which do not show any optical counterpart (all the late-AGB stars plus IRAS 09024-5019), neither those objects classified as of unknown size/morphology in Table 1 . Note that this class of objects may actually contain a mixture of round/elliptical and bipolar sources, as deduced from recent observations of similar objects made with HST (although they could look point-like sources in ground-based CCD images) (e.g. Ueta et al. 2000).

The remaining sources in the sample were classified as round/elliptical or bipolar depending on the morphological properties derived from ground-based or HST observations (see again Table 1 ).

If we concentrate our attention now on the ten sources showing $\mathrm{H}_{2}$ emission in our sample we can see that morphological information is available for six of them, out of which five are classified as bipolar in Table 1, while only one is considered to be round/elliptical. 

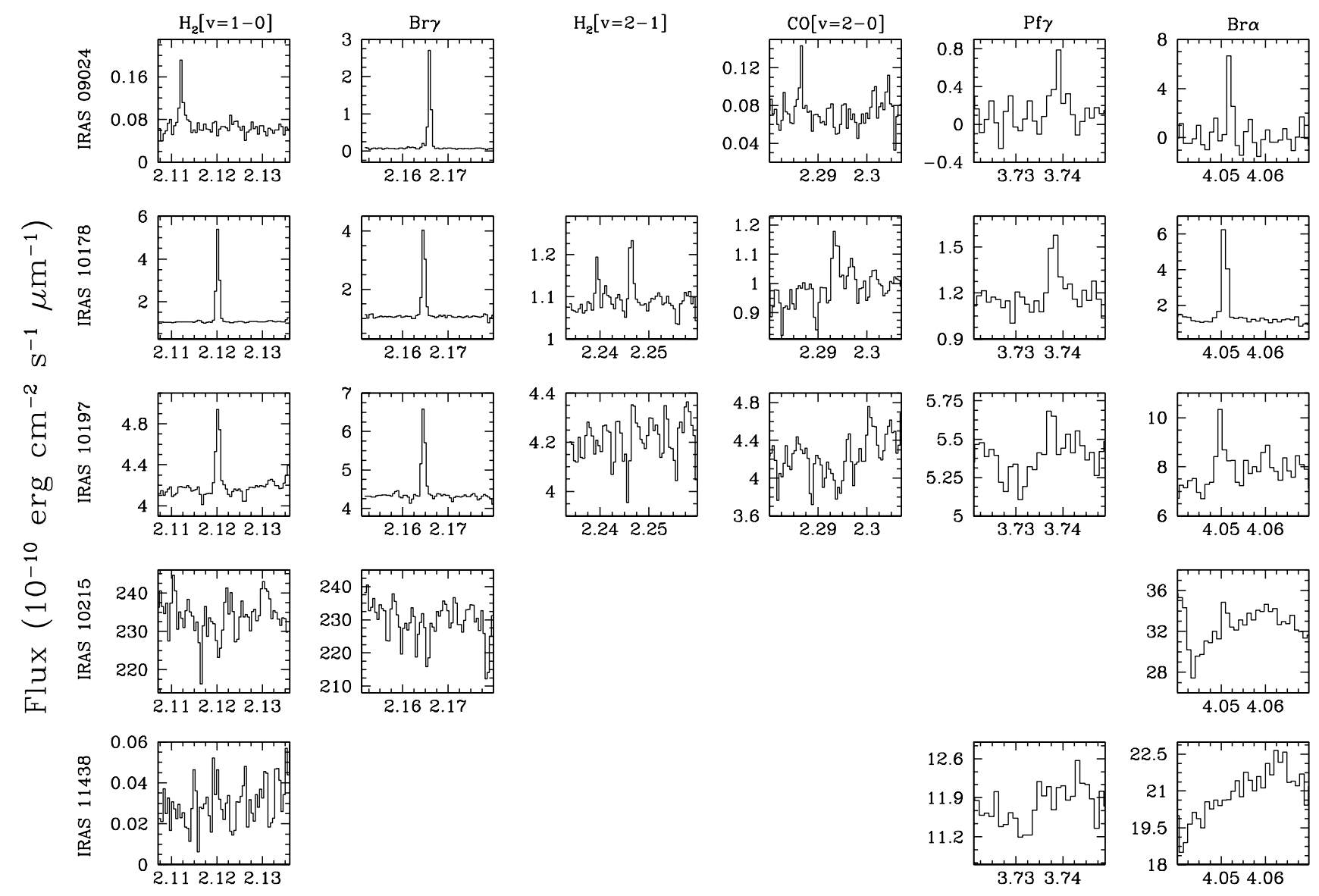

\section{Wavelength $(\mu \mathrm{m})$}

Fig. 1. c) Near infrared spectra of the sources detected with IRSPEC (continued). The best $\mathrm{H}_{2}[v=1 \rightarrow 0 \mathrm{~S}(1)]$ and $\mathrm{Br} \gamma$ spectra for IRAS 10178-5958 correspond to the 1994 run.

However, among the bipolar objects in our sample (eight in total), there are three of them which were not detected in $\mathrm{H}_{2}$. This argues against the idea of a one to one correlation between $\mathrm{H}_{2}$ emission and bipolarity.

In summary, our main conclusion is that although there seems to be an association between bipolarity and the presence of $\mathrm{H}_{2}$ emission the correlation is not so strong as previously reported by Kastner et al. (1996), who derived this conclusion from the observations of well evolved PNe.

\subsection{The excitation mechanism of $\mathrm{H}_{2}$}

Molecular hydrogen can be excited either in shocks or by UV photons (Burton 1992). In order to investigate which of these two possible mechanisms is responsible for the emission observed in the objects included in our sample it is useful to compare the relative strength of the $\mathrm{H}_{2}[v=$ $1 \rightarrow 0 \mathrm{~S}(1)]$ and $\mathrm{H}_{2}[v=2 \rightarrow 1 \mathrm{~S}(1)]$ lines. From the analysis of this diagnostic ratio it is possible to determine whether the excitation is produced by shocks (Burton et al. 1992) or by fluorescence through the absorption of UV photons coming from the central star (Black \& van Dishoeck 1987;
Sternberg \& Dalgarno 1989). For moderate UV radiation fields and gas densities lower than $10^{5} \mathrm{~cm}^{-3}$, shockexcited emission is expected to be associated to flux ratios $\mathrm{H}_{2}[v=1 \rightarrow 0 \mathrm{~S}(1) / v=2 \rightarrow 1 \mathrm{~S}(1)] \sim 10$ while in the case of emission induced by fluorescence a value of $\sim 2$ (Aspin et al. 1993; Ramsay 1993; Burton et al. 1998) is predicted. In Table 3 we show the ratios derived for 9 out of the 10 postAGB stars in the sample detected in molecular hydrogen for which data is available. The estimated uncertainty is $\sim 20 \%$. For those objects in which the $\mathrm{H}_{2}[v=2 \rightarrow 1 \mathrm{~S}(1)]$ line was not detected lower limits are given.

As we can see in Table 3 a wide range of values is obtained. If the above mentioned models are applied to the stars in our list we deduce that shock-excited $\mathrm{H}_{2}$ emission must be the dominant excitation mechanism in at least three of the objects in our sample with a clearly marked bipolar morphology: IRAS 10178-5958, IRAS 16594-46456 (both of uncertain spectral type) and IRAS 17150-3224 (G-type central star) as well as in two other objects of unknown morphology: IRAS 14331-6435 and IRAS 17311-4924 (both with Btype central stars), while fluorescence excitation seems to be the prevalent excitation mechanism in the Stingray 

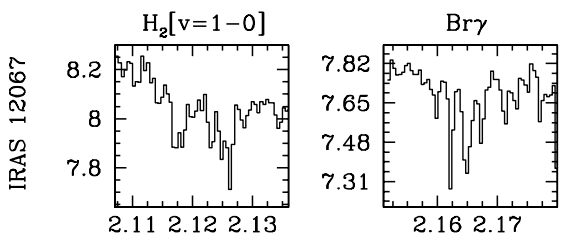

$\mathrm{H}_{2}[\mathrm{v}=2-1] \quad \mathrm{CO}[\mathrm{v}=2-0]$

Pf $\gamma$
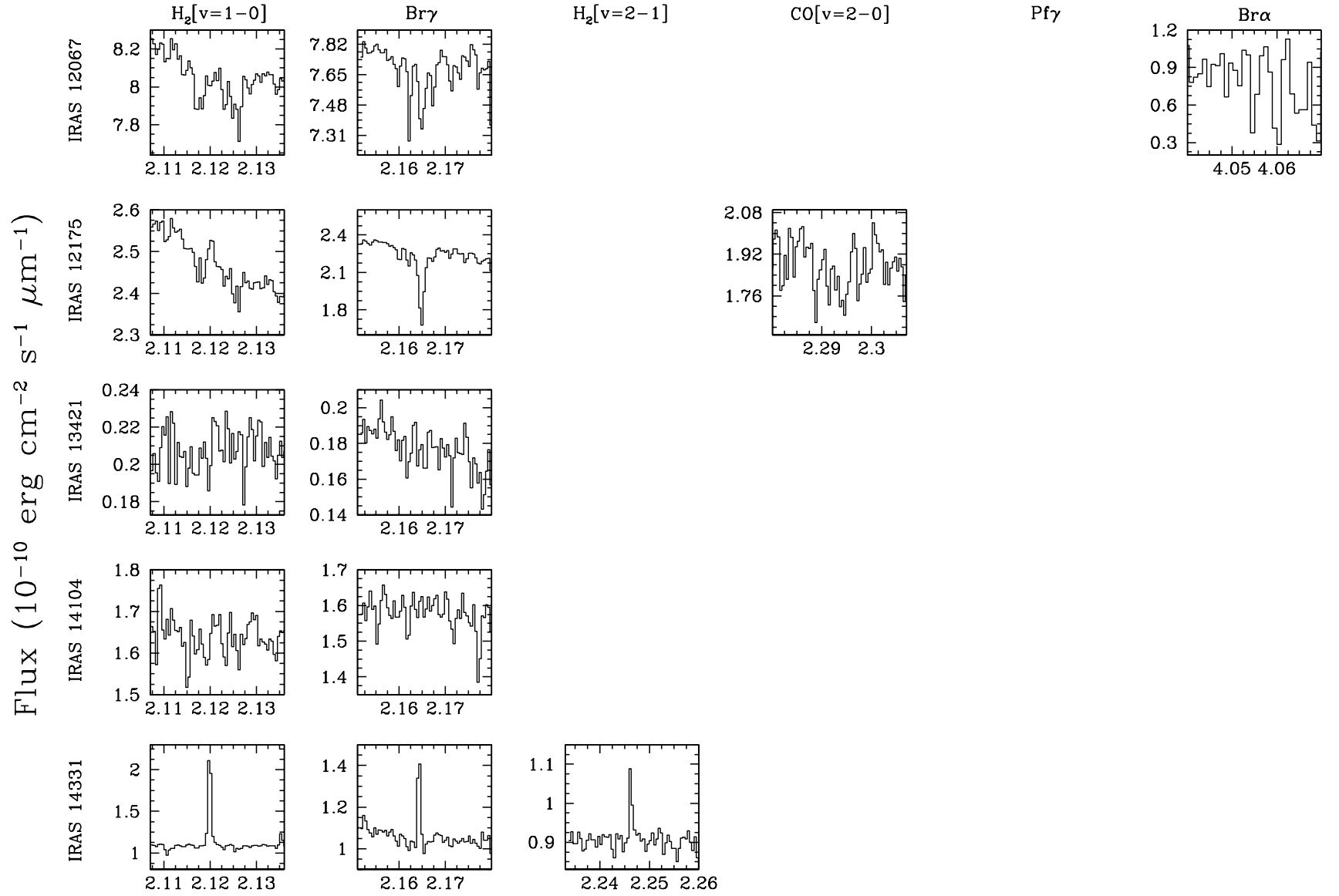

Wavelength $(\mu \mathrm{m})$

Fig. 1. d) Near infrared spectra of the sources detected with IRSPEC (continued).

Nebula (IRAS 17119-5926), which is a very young PN showing an incipient bipolar morphology. For the remaining objects the lower limits found are ambiguous to support either fluorescence or shock excitation.

From the results obtained, there seems to be a prevalence of shock excitation in those objects showing the most extreme bipolar morphologies. However, the results are not conclusive since the statistics are very poor. There is also no evident connection between the excitation mechanism derived from the analysis of the $\mathrm{H}_{2}[v=1 \rightarrow 0$ $\mathrm{S}(1) / v=2 \rightarrow 1 \mathrm{~S}(1)]$ ratio and the spectral type of the central star in which molecular hydrogen has been detected. However, in this latter analysis we must be careful since it is well known that episodic mass loss during the postAGB phase can induce significant spectral changes which may alter for some time the observable spectral type and, thus, taking the spectral type as an indicator of the evolutionary stage might be not the best choice (Bobrowsky et al. 1998; García-Lario et al. 2002).

In addition, an important caveat needs to be considered when interpreting the data in Table 3 . This is the fact that the models above mentioned lose their validity at very high densities $\left(n_{\mathrm{e}}>10^{5} \mathrm{~cm}^{-3}\right)$ and we know that some of the post-AGB stars and transition objects under study are surrounded by regions of these very high densities. In this case, collisional de-excitation of fluorescence-excited levels ("collisional fluorescence") might become important, increasing the $v=0$ and 1 populations over the pure fluorescent values, leading to higher $\mathrm{H}_{2}[v=1 \rightarrow 0 \mathrm{~S}(1) / v=2 \rightarrow 1$ $\mathrm{S}(1)$ ] ratios (Sternberg \& Dalgarno 1989; Burton et al. 1990) which could mimic shock excitation.

In order to address the problem from a different point of view it is also useful to study the detailed spatial distribution of the molecular hydrogen emission and compare this, whenever possible with the spatial distribution observed in the light of $\mathrm{Br} \gamma$.

The $\mathrm{H}_{2}[v=1 \rightarrow 0 \mathrm{~S}(1)] / \mathrm{Br} \gamma$ ratio has recently found to be correlated both with the evolutionary stage and the degree of bipolarity in a sample of well known bipolar PNe (Guerrero et al. 2000). Bipolar PNe with small size, poorly defined rings and bright central stars exhibit small $\mathrm{H}_{2}[v=1 \rightarrow 0 \mathrm{~S}(1)] / \operatorname{Br} \gamma$ ratios $(\sim 0.1-0.5)$ while those with large, well defined rings and faint central stars showed larger $\mathrm{H}_{2}[v=1 \rightarrow 0 \mathrm{~S}(1)] / \operatorname{Br} \gamma$ ratios $(>5)$.

Guerrero et al. (2000) suggest that the excitation of the molecular hydrogen in each of these groups turns from fluorescence excitation at the early stages of $\mathrm{PNe}$ to shock-excited emission as the bipolar PNe evolve. This 

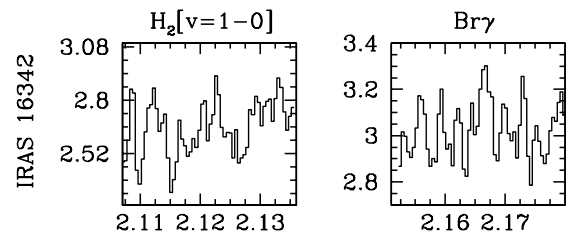

$\mathrm{H}_{2}[\mathrm{v}=2-1]$

$\mathrm{CO}[\mathrm{v}=2-0]$

$\operatorname{Pf} \gamma$
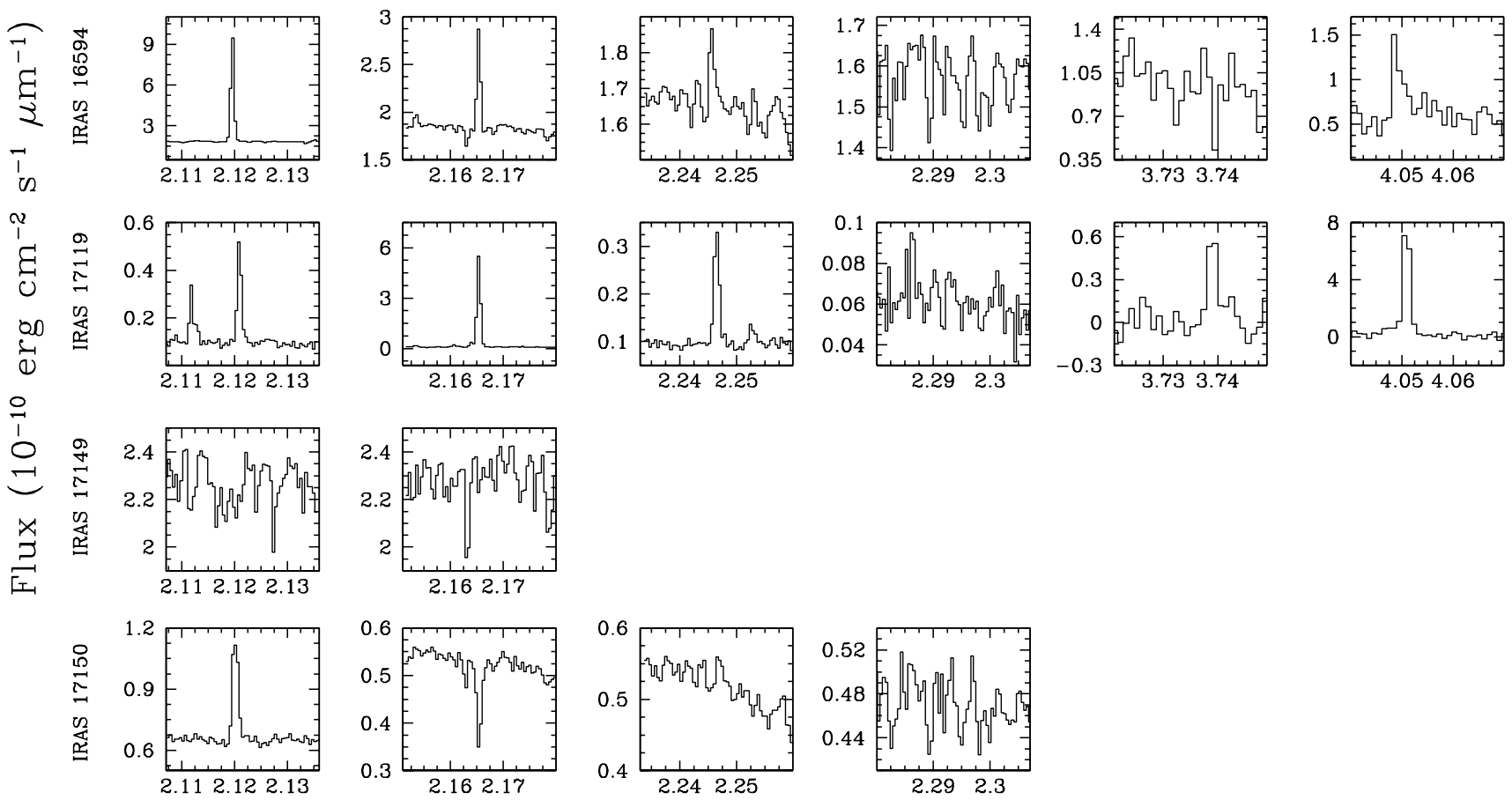

\section{Wavelength $(\mu \mathrm{m})$}

Fig. 1. e) Near infrared spectra of the sources detected with IRSPEC (continued). The best $\mathrm{H}_{2}[v=1 \rightarrow 0 \mathrm{~S}(1)]$ spectra for IRAS 16594-4656 and IRAS 17150-3224 correspond to the 1994 run.

is supported by the different spatial distribution of the observed molecular hydrogen emission.

$\mathrm{H}_{2}$-dominated bipolar PNe are found to show different morphological and physical properties. Their central regions have low surface brightness, in agreement with their low densities while $\mathrm{Br} \gamma$-dominated bipolar PNe have a high surface brightness and larger densities.

If this effect is interpreted as an evolutionary sequence (Balick 1987) then the less evolved bipolar PNe with molecular hydrogen emission would start their evolution as $\mathrm{Br} \gamma$-dominated becoming later $\mathrm{H}_{2}$-dominated.

Note, however, that this scenario does not apply to all PNe. Well evolved, low-mass density-bounded round/elliptical PNe are not expected to preserve hydrogen in the form of molecules since most (if not all) of the hydrogen in the circumstellar envelope becomes ionized as the ionization front passes through the gas. These PNe will end their lives as $\mathrm{Br} \gamma$-dominated, unlike the group of $\mathrm{PNe}$ with more massive progenitors. Those with more massive progenitors are expected to develop ionization-bounded nebulae, where a substantial fraction of the mass ejected will never become ionized. This enables the detection of neutral material in the circumstellar gas even at a very late stage as PNe, provided the UV radiation field from the central star is hard enough to produce fluorescence excitation. In the subgroup of bipolar PNe, the shielding provided by the dense equatorial regions that collimate the outflow from the UV photons escaping from the central star would also favor the preservation of hydrogen in molecular state. This would explain the high detection rate of molecular hydrogen emission in bipolar type I PNe, which are suspected to be the result of the evolution of stars with large progenitor masses. In this latter case, molecular hydrogen can also be excited by shocks formed in the high velocity outflow.

Applying the above considerations to the analysis of the molecular hydrogen emission observed in precursors of PNe it would be natural to expect fluorescence to be activated when the radiation coming from the central postAGB star becomes hard enough to produce a significant number of UV photons as the central star moves in the HR diagram towards hotter effective temperatures in its way to becoming a PN. On the other hand, the interaction of the fast post-AGB wind with the slow-moving material ejected during the previous AGB phase would favor the formation of shocks, especially in those objects showing 

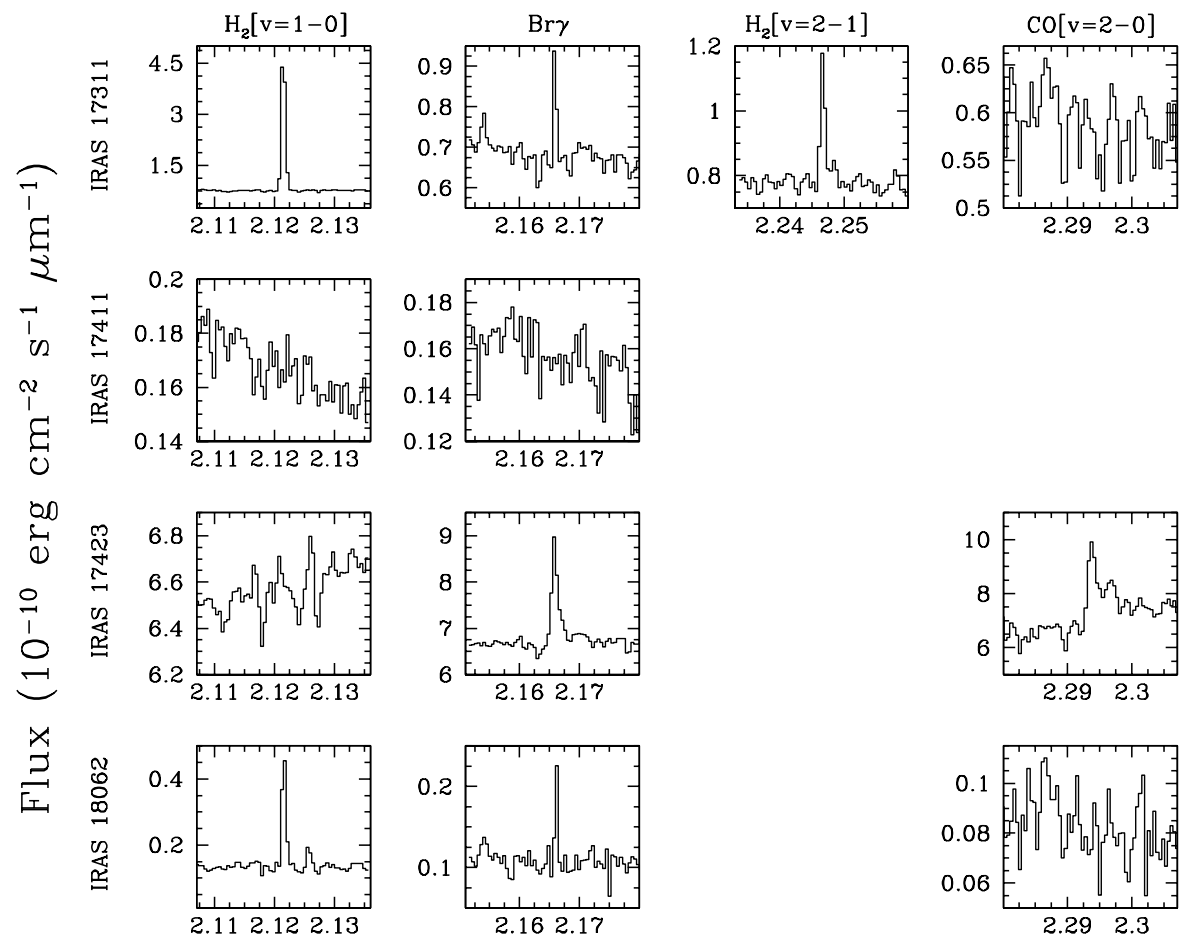

$\operatorname{Br} \alpha$
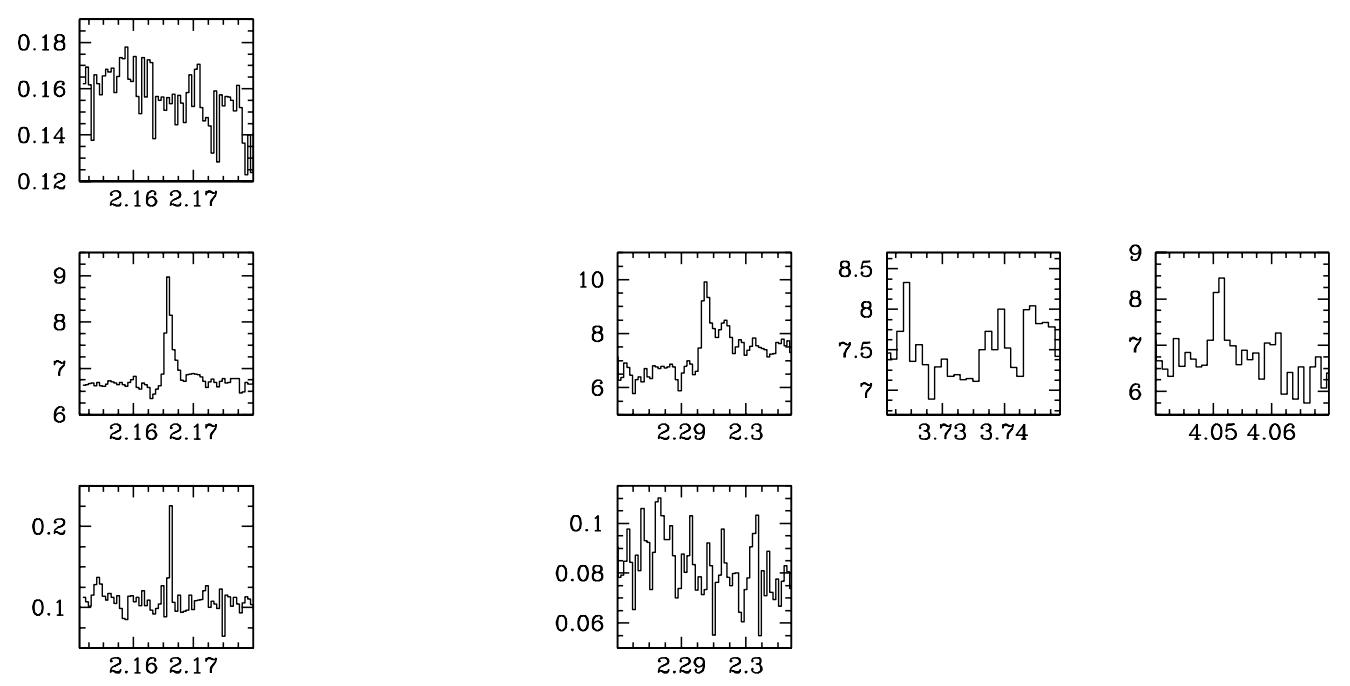

\section{Wavelength $(\mu \mathrm{m})$}

Fig. 1. f) Near infrared spectra of the sources detected with IRSPEC (continued).

a strong collimation induced by the presence of a thick equatorial disk or torus, in the process of developing a bipolar morphology.

In order to check whether this scenario is consistent with the data obtained on the stars in our sample we have derived the $\mathrm{H}_{2}[v=1 \rightarrow 0 \mathrm{~S}(1)] / \operatorname{Br} \gamma$ ratios for the objects that exhibit $\mathrm{H}_{2}$ emission. The results obtained are presented in Table 3, where we can see that, as for the $\mathrm{H}_{2}[v=1 \rightarrow 0 \mathrm{~S}(1) / v=2 \rightarrow 1 \mathrm{~S}(1)]$ ratio, a wide range of values is found (the quoted values, not corrected for extinction, again have an estimated uncertainty of $\sim 20 \%$ ).

Only four sources (out of ten) are found to be $\operatorname{Br} \gamma$-dominated. These are: IRAS 06556+1623, IRAS 07027-7934, IRAS 10197-5750 and IRAS 17119-5926. Detailed morphological information exists for the latter three sources. IRAS 07027-7934 is known to be a very young PN showing a round/elliptical shape. The bipolar morphology is only incipient in IRAS 17119-5926 which otherwise would look also quite round/elliptical. The only source in this short list with a marked bipolar morphology is IRAS 10197-5750 (Sahai et al. 1999), which is seen almost edge-on. This source has recently been imaged with HST (NICMOS) in the light of $\mathrm{H}_{2}[v=1 \rightarrow 0 \mathrm{~S}(1)]$ (Sahai et al. 2000) and the spatial distribution of the emission in this line is found to be very similar to the distribution of scattered light in the bright lobes, suggesting that fluorescence excitation is the main mechanism contributing to the overall observed emission. However, a clear enhancement of emission is observed at two localized point-symmetric regions distant from the central star which unfortunately have not been covered by our slit (the slit orientation was $90^{\circ}$ and its bipolar axis is orientated $\sim+20^{\circ}$ ), which we interpret as shock-excited emission probably originated by the interaction of the high velocity post-AGB wind with the slow moving old AGB shell. Interestingly, the four $\operatorname{Br} \gamma$-dominated sources show $\mathrm{H}_{2}[v=1 \rightarrow 0 \mathrm{~S}(1) / v=2 \rightarrow 1 \mathrm{~S}(1)]$ ratios compatible with fluorescence excitation (see Table 3 ).

Two other sources in our list have also been imaged in the light of $\mathrm{H}_{2}[v=1 \rightarrow 0 \mathrm{~S}(1)]$ with HST (NICMOS). These are IRAS 10178-5958 (Sahai et al. 2000) and IRAS 17150-3224 (Su et al. 2000) belonging to the group of $\mathrm{H}_{2}$-dominated sources in our sample.

In both cases, the spatial distribution of the molecular hydrogen emission suggests a shock-excited origin. In IRAS 10178-5958, the molecular hydrogen emission comes mainly from the walls of the bipolar lobes (GarcíaLario et al. 1999b), while in IRAS 17150-3224 the bulk of the detected emission arises in an expanding molecular torus surrounding the central star (Weintraub et al. 1998) although several clumps of molecular hydrogen emission are also detected in the outer regions of the bipolar lobes according to $\mathrm{Su}$ et al. (2000). A similar spatial distribution was also found by Sahai et al. (1998) in the case of AFGL 2688, where the emission observed was also interpreted as due to shock excitation.

Unfortunately, none of the four other $\mathrm{H}_{2}$-dominated sources in Table 3 have been imaged in molecular hydrogen with HST. Three of them (namely IRAS 14331-6435, IRAS 17311-4924 and IRAS 18062+2419) are sources 
Table 3. Flux ratios observed in the sources showing molecular hydrogen emission and prevalent excitation mechanism tentatively inferred from the data.

\begin{tabular}{lcccl}
\hline \hline Object & $\mathrm{H}_{2}[v=1 \rightarrow 0 \mathrm{~S}(1)]^{1}$ & $\mathrm{H}_{2}[v=1 \rightarrow 0 \mathrm{~S}(1)] / \mathrm{H}_{2}[v=2 \rightarrow 1 \mathrm{~S}(1)]$ & $\mathrm{H}_{2}[v=1 \rightarrow 0 \mathrm{~S}(1)] /$ Br $\gamma$ & Excitation \\
\hline IRAS 06556+1623 & 3.4 & $>1.7$ & 0.05 & Fluorescence \\
IRAS 07027-7934 & 2.4 & $>1.3$ & 0.18 & Fluorescence \\
IRAS 10178-5958 & 37.1 & 26.5 & 1.50 & Shock \\
IRAS 10197-5750 & 8.9 & $>2.6$ & 0.40 & Fluorescence* \\
IRAS 14331-6435 & 10.1 & 8.4 & 2.97 & Shock \\
IRAS 16594-4656 & 62.7 & 33 & 6.82 & Shock \\
IRAS 17119-5926 & 4.1 & 1.5 & 0.09 & Fluorescence \\
IRAS 17150-3224 & 5.5 & $>7.8$ & 2 & Shock \\
IRAS 17311-4924 & 36.3 & 11.3 & 18.2 & Shock \\
IRAS 18062+2410 & 3.0 & -3 & 3.75 & Shock? \\
\hline
\end{tabular}

${ }^{1}$ Emission line flux in units of $10^{-14} \mathrm{erg}^{\mathrm{cm}^{-2}} \mathrm{~s}^{-1}$.

${ }^{2} \mathrm{Br} \gamma$ detected in absorption.

${ }^{3}$ Not observed in the $\mathrm{H}_{2}[v=2 \rightarrow 1 \mathrm{~S}(1)]$ line.

* Shock-excited emission can be present in the outer lobes as deduced from recent HST images but they were not covered by our slit.

not spatially resolved from ground-based observatories. Only IRAS 16594-4656 was found to be extended from the ground in optical CCD images and it was later imaged with HST in the optical showing an extended, complex multi-polar morphology (García-Lario et al. 1999a; Hrivnak et al. 1999). All of them show large $\mathrm{H}_{2}[v=1 \rightarrow 0$ $\mathrm{S}(1) / v=2 \rightarrow 1 \mathrm{~S}(1)]$ ratios which are consistent with shock excitation.

The emission seems to be extended only in the case of IRAS $10178-5958$ and IRAS $17119-5926$ but we did not find any spatial dependence of the measured $\mathrm{H}_{2}[v=1 \rightarrow 0$ $\mathrm{S}(1) / v=2 \rightarrow 1 \mathrm{~S}(1)]$ ratios along the nebulae. This confirms shock excitation as the prevalent mechanism of excitation of the molecular hydrogen in IRAS 10178-5958 throughout its whole nebular extension. On the other hand, fluorescence excitation seems to be the mechanism through which molecular hydrogen gets excited in the case of IRAS 17119-5926. For IRAS 17119-5926, 2D spectra of both $\mathrm{H}_{2}$ lines were taken with different (perpendicular) slit orientations in 1993 and 1994. Remarkably, the molecular hydrogen emission seems to be quite homogeneous independent of the slit orientation considered and extends well beyond the limits of the $\operatorname{Br} \gamma$ emission. This suggests that most of the emitting molecular hydrogen is located outside the small ionized region of this very young PN, in the still neutral outer shell.

In summary, when we integrated all the flux received in each line, we obtain high $\mathrm{H}_{2}[v=1 \rightarrow 0 \mathrm{~S}(1) / v=2 \rightarrow 1$ $\mathrm{S}(1)]$ and $\mathrm{H}_{2}[v=1 \rightarrow 0 \mathrm{~S}(1)] / \operatorname{Br} \gamma$ ratios that are consistent with the prevalence of shock excitation, while low ratios indicate fluorescence as the dominant mechanism for the observed $\mathrm{H}_{2}$ emission. This is supported by the different spatial distribution seen in those objects with existing $\mathrm{H}_{2}$ NICMOS images.
With our small sample and the rest of available data we find different types of sources that exhibit $\mathrm{H}_{2}$ emission during the post-AGB phase. We propose that this scenario could be summarized as follows:

1) In some cases, especially strongly bipolar sources with known early spectral types, there must be a contribution from the two excitation mechanisms in the observed emission (e.g. IRAS 10197-5750). It is very difficult to distinguish the contribution of both excitation mechanisms but it seems clear that the shocked $\mathrm{H}_{2}$ emission is more intense than the fluorescence-excited emission, leading to higher $\mathrm{H}_{2}[v=1 \rightarrow 0 \mathrm{~S}(1) / v=2 \rightarrow 1$ $\mathrm{S}(1)]$ and $\mathrm{H}_{2}[v=1 \rightarrow 0 \mathrm{~S}(1)] / \mathrm{Br} \gamma$ ratios.

2) The $\mathrm{H}_{2}$ emission that comes from bipolar sources with later than A spectral types (e.g. AFGL 2688 and IRAS 17150-3224) is shock-excited because the central postAGB star is not hot enough to produce fluorescent emission, which, so it has been demonstrated, is correlated with the evolutionary stage (spectral type) of the central post-AGB star. So the presence of shockexcited emission early in the post-AGB phase must be indicative of the evolutionary morphological stage of the nebulosity that surrounds the central post-AGB star. However, it remains unclear if the early development of marked bipolar morphologies during the postAGB phase could be dependent on the mass of the central progenitor star. For higher masses, the morphological evolution could be faster and the formation of bipolar morphologies is easier; so, therefore, is the detection of shock-excited $\mathrm{H}_{2}$ emission.

3) On the other hand, the fact that $\mathrm{H}_{2}$ emission is detected towards well evolved bipolar PNe could be indicative that the destruction of neutral material around the non-bipolar ones (e.g. IRAS 07027-7934 or even IRAS 17119-3224) can roughly coincide with the 
photoionization of the circumstellar shell. This could indicate that the fluorescence-excited $\mathrm{H}_{2}$ emission detected in non-bipolar sources is observed only during a short period of time in the post-AGB phase. However, in those bipolar objects where the wind is strongly collimated by the presence of an optically thick equatorial disc or torus, the molecular material can be shielded from the ionizing photons coming from the central star.

This entire hypothesis deserves further study to understand better the physical and chemical processes involved during the short transition phase from the AGB phase to the PN stage. At present, the number of known transition objects and the obtaining of high quality data are growing continuously. A detailed spatially resolved spectroscopic analysis of the $\mathrm{H}_{2}$ emission line spectrum in a large sample of planetary nebulae precursors is needed to address these issues fully.

\section{Conclusions}

With the data here presented we have extended the number of post-AGB stars and/or proto-PNe where molecular hydrogen emission has been detected from four to thirteen.

Our results confirm the previous finding by Weintraub et al. (1998) made on a smaller sample of sources that the onset of emission from molecular hydrogen takes place in the post-AGB phase. In many cases this occurs well after the generation of the bipolar structure takes place and before the nebular envelope gets ionized.

In the past it has been suggested that molecular hydrogen emission is a signpost for bipolarity in PNe (Kastner et al. 1996). We find that the detection of molecular hydrogen strongly depends on the excitation mechanism.

The detection of fluorescence-excited molecular hydrogen emission in precursors of PNe is strongly correlated with the evolutionary stage of the central star (spectral type), independent of the morphology of the source considered. The fluorescence excitation of molecular hydrogen emission is produced as a consequence of the absorption of the increasing number of UV photons escaping from the relatively hot, rapidly evolving central post-AGB star by the hydrogen molecules present in the circumstellar shell. As a general trend, we find that fluorescent $\mathrm{H}_{2}$ emission becomes active when the central post-AGB star reaches a certain temperature that corresponds to an A-spectral type.

On the other hand, shock-excited emission is only detected in objects with a marked bipolar morphology, sometimes at a very early stage during the post-AGB phase, when the star is still very cool and, sometimes, localized in the waist and/or in the specific regions of the bipolar lobes (either at the walls, like in IRAS 10178-5958, or at the tips, like in IRAS 10197-5750), where a physical interaction exists between fast- and slow-moving material.

Bipolarity also helps preserving the molecular hydrogen from destruction, favoring its detection in well evolved bipolar PNe. Actually, with very few exceptions, the brightest $\mathrm{H}_{2}$ emission, shock-excited, is detected toward the waist of bipolar PNe (Kastner et al. 1996). The high correlation found between bipolarity and molecular hydrogen emission seems to be just the consequence of the bias induced by this fact.

The diagnostic ratios $\mathrm{H}_{2}[v=1 \rightarrow 0 \mathrm{~S}(1) / v=2 \rightarrow 1 \mathrm{~S}(1)]$ and $\mathrm{H}_{2}[v=1 \rightarrow 0 \mathrm{~S}(1)] / \operatorname{Br} \gamma$ are shown to be very good indicators of the prevalent mechanism of excitation in proto-PNe. Unfortunately, the information on the relative spatial distribution of the different molecular hydrogen lines with respect to the hydrogen recombination lines is still sparse. Without this information we cannot determine the precise localization of shock-excited and fluorescenceexcited regions on individual sources. As we have shown through this paper this is a strong limitation if we want to understand the physical processes which are taking place in this still poorly known transition phase which precedes the formation of $\mathrm{PNe}$. This is especially important to interpret the emission observed in complex objects like IRAS 10197-5750, where both fluorescence- and shockexcited regions may coexist. High spatial resolution images (e.g. as in the past with NICMOS on board HST) in the three emission lines above mentioned for a statistically significant number of transition objects and young PNe will provide this crucial information. This would be an ideal way to test the scenario here proposed.

Acknowledgements. PGL and AM acknowledges support from grant PB97-1435-C02-02 from the Spanish Dirección General de Enseñanza Superior (DGES).

\section{References}

Aspin, C., Schwarz, H. E., Smith, M. G., et al. 1993, A\&A, 278,255

Balick, B. 1987, AJ, 94, 671

Beckwith, S., Gatley, I., \& Persson, S. E. 1978, ApJ, 219, L33

Black, J. H., \& van Dishoeck, E. F. 1987, ApJ, 322, 412

Bobrowsky, M., Sahu, K. C., Parthasarathy, M., \& GarcíaLario, P. 1998, Nature, 392, 469

Bujarrabal, V., Gómez-González, J., Bachiller, R., \& MartínPintado, J. 1988, A\&A, 204, 242

Burton, M. G. 1992, Aust. J. Phys., 45, 463

Burton, M. G., Hollenbach, D. J., \& Tielens, A. G. M. M. 1990, ApJ, 365, 620

Burton, M. G., Hollenbach, D. J., \& Tielens, A. G. M. M. 1992, ApJ, 399, 563

Burton, M. G., Howe, J. E., Geballe, T. R., \& Brand, P. W. J. L. 1998, Publ. Astron. Soc. Aust., 15, 194

Cernicharo, J., Guélin, M., \& Kahane, C. 1999, A\&AS, 142, 181

Engels, D. 1979, A\&AS, 36, 337

Engels, D., \& Lewis, B. M. 1996, A\&AS, 116, 117

García-Lario, P., Manchado, A., Pottasch, S. R., Suso, J., \& Olling, R. 1990, A\&AS, 82, 497

García-Lario, P., Manchado, A., Riera, A., Mampaso, A., \& Pottasch, S. R. 1991, A\&A, 249, 223

García-Lario, P., Manchado, A., Parthasarathy, M., \& Pottasch, S. R. 1994, A\&A, 285, 179 
García-Lario, P., Manchado A., Parthasarathy, M., Pych, W., \& Pottasch, S. R. 1997a, A\&AS, 126, 479

García-Lario, P., Parthasarathy, M., de Martino D., et al. 1997b, A\&A, 326, 1103

García-Lario, P., Manchado, A., Ulla, A., \& Manteiga, M. 1999a, ApJ, 513, 941

García-Lario, P., Riera, A., \& Manchado, A. 1999b, ApJ, 526, 854

García-Lario, P., Suarez, O., Manchado, A., \& Manteiga, M. 2002 , in preparation

Gatley, I., Depoy, D. L., \& Fowler, A. M. 1988, Science, 242, 1264

Guerrero, M. A., Villaver, E., Manchado, A., García-Lario, P., \& Prada, F. 2000, ApJS, 127, 125

Hora, J. L., Latter, W. B., \& Deutsch, L. K. 1999, ApJS, 124, 195

Hrivnak, B. J., Kwok, S., \& Su, K. Y. L. 1999, ApJ, 524, 849

Hrivnak, B. J., Volk, K., \& Kwok, S. 2000, ApJ, 535, 275

Huggins, P. J., \& Healy, A. 1989, ApJ, 346, 201

Huggins, P. J., Bachiller, R., Cox, P., \& Forveille, T. 1996, A\&A, 315, 284

Kastner, J. H., Weintraub, D. A., Gatley, I., Merril, K. M., \& Probst, R. G. 1996, ApJ, 462, 777

Kwok, S. 1994, PASP, 106, 344

Latter, W. B., Kelly, D. M., Hora, J. L., \& Deutsch, L. K. 1995 , ApJS, 100, 159

Likkel, L., Forveille, T., Omont, A., \& Morris, M. 1991, A\&A, 246, 153

Lindqvist, M., Schöier, F. L., Lucas, R., \& Olofsson, H. 2000, A\&A, 361, 1036

Loup, C., Forveille, T., Omont, A., \& Paul, J. F. 1993, A\&AS, 99, 291

Manchado, A., Pottasch, S. R., García-Lario, P., Esteban, C., \& Mampaso, A. 1989a, A\&A, 214, 139

Moorwood, A., Biereichel, P., Finger, G., et al. 1986, Messenger, 44, 19

Neri, R., Kahane, C., Lucas, R., Bujarrabal, V., \& Loup, C. 1998, A\&AS, 130, 1

Nyman, L.-A., Hall, P. J., \& Olofsson, H. 1998, A\&AS, 127, 185

Oudmaijer, R. D., Waters, L. B. F. M., van der Veen, W. E. C. J., \& Geballe, T. R. 1995, A\&A, 299, 69
Pottasch, S. R. 1980, A\&A, 89, 336

Pottasch, S. R. 1992, A\&ARv, 4, 215

Ramsay, S. K. 1993, Ph.D. Thesis, University of Edinburgh, UK

Riera, A., García-Lario, P., Manchado, A., Pottasch, S. R., \& Raga, A. C. 1995, A\&A, 302, 137

Sahai, R., Hines, D., Kastner, J. H., et al. 1998, ApJ, 492, L163

Sahai, R., Zijlstra, A., Bujarrabal, V., \& te Lintel Hekkert, P. 1999, AJ, 117, 1408

Sahai, R., Su, K. Y. L., Kwok, S., Dayal, A., \& Hrivnak, B. J. 2000, in Asymmetrical Planetary Nebulae II: from Origins to Microstructures, ASP Conf. Ser., 199, ed. J. H. Kastner, N. Soker, \& S. Rappaport, 167

Sternberg, A., \& Dalgarno, A. 1989, ApJ, 338, 197

Su, K. Y. L., Kwok, S., Hrivnak, B. J., \& Sahai, R. 2000, in Asymmetrical Planetary Nebulae II: from Origins to Microstructures, ASP Conf. Ser., 199, ed. J. H. Kastner, N. Soker, \& S. Rappaport, 163

Sylvester, R. J., Kemper, F., Barlow, M. J., et al. 1999, A\&A, 352,587

te Lintel Hekkert, P. 1991a, A\&A, 248, 209

te Lintel Hekkert, P., Caswell, J. L., Habing, H. J., Haynes, R. F., \& Norris, R. P. 1991b, A\&AS, 90, 327

te Lintel Hekkert, P., \& Chapman, J. M. 1996, A\&A, 119, 459

Treffers, R. T., Fink, U. F., Larson, H. L., \& Gautier, N. T. 1976, ApJ, 209, 793

Ueta, T., Meixner, M., \& Bobrowsky, M. 2000, ApJ, 528, 861

Wallerstein, G., \& Knapp, G. R. 1998, ARA\&A, 36, 369

Waters, L. B .F. M., Beintema, D. A., Cami, J., et al. 1999, in The Universe as seen by ISO, ed. P. Cox, \& M. F. Kessler, ESA-SP 427, 219

Webster, B. L., Payne, P. W., Storey, J. W. V., \& Dopita, M. A. 1988, MNRAS, 235, 533

Weintraub, D. A., Huard, T., Kastner, J., \& Gatley, I. 1998, ApJ, 509, 728

Zijlstra, A. A., te Lintel Hekkert, P., Pottasch, S. R., et al. 1989, A\&A, 217, 157

Zijlstra, A. A., Gaylard, M. J., te Lintel Hekkert, P., et al. 1991, A\&A, 243, L9

Zuckerman, B., \& Gatley, I. 1988, ApJ, 324, 501 